\title{
Emotional Support and Well-Being of Adults with Congenital Heart Disease (ACHD): Contributions of a Specialist Nursing Service
}

\author{
Richard Hatchett ${ }^{1}$, Susan McLaren ${ }^{2}$, Fiona Kennedy ${ }^{3 *}$ \\ ${ }^{1}$ Former Deputy Head of School, The Royal Marsden School, The Royal Marsden NHS Foundation Trust, \\ London, UK \\ ${ }^{2}$ Former Emeritus Professor, Faculty of Health and Social Care, London South Bank University, London, UK \\ ${ }^{3}$ Adults with Congenital Heart Disease (ACHD) Nurse Specialist, Barts Healthcare NHS Trust, London, UK \\ Email: "Fiona.Kennedy@bartshealth.nhs.uk
}

Received 11 June 2015; accepted 26 December 2015; published 29 December 2015

Copyright $@ 2015$ by authors and Scientific Research Publishing Inc.

This work is licensed under the Creative Commons Attribution International License (CC BY).

http://creativecommons.org/licenses/by/4.0/

\section{(c) (i) Open Access}

\begin{abstract}
Living with a congenital heart condition can require a daunting and uncertain patient journey during which the support of a specialist nursing service is vital. A descriptive, cross-sectional evaluation completed over two years, utilised a postal questionnaire to investigate patient's satisfaction with aspects of service. Qualitative responses to open questions on satisfaction with emotional support $(n=103)$ and contributions to well-being $(n=90)$ were analysed using a framework approach which identified eight themes. Professional knowledge and expertise, service accessibility, caring attributes, enabling patients to cope with anxiety, depression and meeting changing needs contributed positively to both patient well-being and emotional support. Family support was influential on emotional support alone, whilst mediating medical liaison exerted an impact on well-being by enhancing feelings of security. In conclusion, the specialist nursing service contributed positively to well-being and emotional support of patients and thereby to selective aspects of continuity of care.
\end{abstract}

\section{Keywords}

Adult Congenital Heart Disease (ACHD)\#, Specialist Nurses, Emotional Support, Well-Being, Patient Evaluation

\footnotetext{
"Corresponding author.

"Please note recent changes in usage regarding the terminology/acronym for this group of patients from Grown Up Congenital Heart Disease (GUCH) to ACHD patients.
}

How to cite this paper: Hatchett, R., McLaren, S. and Kennedy, F. (2015) Emotional Support and Well-Being of Adults with Congenital Heart Disease (ACHD): Contributions of a Specialist Nursing Service. Open Journal of Nursing, 5, 1133-1141. 


\section{Introduction}

Over the last five decades, advances in cardiac surgery, anaesthetics, medical and intensive care resulting in greater life expectancy have led to an expansion in the populations of ACHD patients in Europe (1.2 million) and the USA (1 million) [1] [2]. Delivering a specialist nursing service for increasing numbers of adults with complex physical, emotional and social needs arising from the severity of their congenital heart disorder, many of whom need lifelong care due to residual lesions and vascular problems, poses a number of challenges for health care systems [2]-[6]. Services now need to provide for changing needs across the adult lifespan. This includes meeting specific requirements imposed by pregnancy, facilitating transfer of young people to adult services, supporting adjustment to situations which give rise to changing healthcare needs (e.g. palliative care) and maximising independence in activities of daily living, all of which requires co-ordination of services across professional and organisational boundaries [3] [7]-[11]. Specialist nursing roles vary across clinical specialities, but can encompass facilitating access to key contacts for support, initiating nurse-led clinics, providing a telephone advisory service, enabling patients' and families' understanding of the condition, conducting pharmacotherapy rescue work, education, physical assessment, together with improving and leading service development [8]-[10] [12]. Expansion in the ACHD population and its changing needs has led to a reappraisal of specialist roles and services, for example utilising a network model linked to national standards which also consider different levels of specialist role development and competencies [13]. Innovation in roles and services need to be informed by the patient experience [14], a driver and motivating force for this service evaluation.

Many ACHD patients have reported difficulties in areas of daily life relating to employment, education, participation in sport, driving and insurability [11] which can impact negatively on quality of life [15]-[17]. Low exercise capacity and medical restrictions are significant predictors for behavioural and emotional problems [17] and concerns have arisen about emotional and mental health issues of living with CHD [18] [19]. Anxiety and depression have been reported, arising from perceptions of financial strain and low levels of social support [19] [20] which can impact negatively on well-being. Well-being relates to individuals' perceptions that life is going well, encompassing "the presence of positive emotions and moods (contentment/happiness) and absence of negative emotions/moods (depression/anxiety)" [21]. It can be defined as a balance point between an individual's pool of psychological, social and physical resources and challenges to these resources [22]. Diverse aspects of well-being include physical, economic, social, emotional and psychological domains.

Little is known of ACHD patients' experiences of nursing interventions which contribute to well-being and emotional support. Systematic reviews investigating specialist nursing roles more widely noted limited evidence for an impact on overall quality of life [23]-[25] although a short-term impact on mental health related quality of life had been reported [26]. Other studies reported high levels of service user's satisfaction with physical care, emotional support and advice for a range of specialist and advanced nursing roles (cancer, transplants, inflammatory bowel disease, cystic fibrosis): the importance of promoting wellness, self-management skills, preparedness for treatment, symptom management, access, efficiency and referral were also emphasised, as was being known to the family and demonstrating kindness [27] [28]. Specialist cancer nurses have been shown to exert positive effects on emotional functioning and the importance of providing appropriate information, supporting families and carers and acting as an intermediary with doctors has been identified [29]. Service users' perceptions of continuity of care emphasise service accessibility, communication, information transfer and relational continuity with a single health professional [30]. Collaboration by specialist nurses with other services, information provision and adjusting to service users' changing needs over time can also contribute to different dimensions of continuity of care, although this has not been investigated with regard to ACHD services [31] [32].

Sparse literature relating to patients' experiences of ACHD specialist nursing services and the need to consider current provision in the light of changing requirements [2]-[11] provided a driving force for this service evaluation. Quantitative findings [33] demonstrated high levels of satisfaction with different elements of care relating to information provision, time available to discuss needs, self-management, symptom distress, emotional support and well-being, and also revealed patients' highest ranked priorities for care were the provision of timely information and advice. Qualitative findings reveal reasons underpinning numerical scores and provide valuable insights into the aspects of service which are highly valued and those needing improvement.

\section{Methods}

This paper presents selective qualitative findings of a wider study [33] in which the overall aim was to evaluate 
ACHD patients' experiences and satisfaction with the delivery of a specialist nursing service and to make recommendations for service development and improvement. With regard to the findings below, the objectives were to explore reasons for patients' satisfaction with emotional support and contribution of the nursing service to their well-being. A final objective was to make recommendations for service delivery with regard to emotional support and well-being.

\subsection{Design and Setting}

A descriptive, cross-sectional service evaluation [33] utilising survey principles was implemented over two years in an NHS Foundation Trust in London. An ACHD specialist nursing service was delivered to a diverse population with needs arising from congenital heart disease and associated comorbid conditions including learning disability. The service was delivered through nurse-led clinics, one of which focussed entirely on the needs of pregnant women. Patients were also seen within a multidisciplinary clinic setting.

\subsection{Ethical Issues}

Following review by the Local Research Ethics Committee, governance approval was granted for a service evaluation. Patients were informed in writing about the purpose of the study, assured that participation was entirely voluntary and that anonymity would be protected.

\subsection{Data Collection}

Patient data were collected utilising a postal questionnaire in which closed questions measured levels of agreement relating to satisfaction with/contributions to aspects of nursing care, with follow up open questions requesting respondents to provide reasons for their ratings [33]. This paper focuses solely on the qualitative findings arising from freehand responses to open questions on respondents' reasons for satisfaction with emotional support and contributions to well-being. Two mailings of anonymised questionnaires were sent at 4 - 6 week intervals to patients $(n=1021)$, achieving an overall unit response rate of $33 \%(n=340)$. Of these, $n=103$ patients provided responses to the open question on emotional support and $n=90$ to that on well-being. Across both questions, respondent's ages ranged from 16 - 70 years, the majority of White British ethnicity (82\%). Female gender predominated, but fewer female respondents (62\%) answered the question on emotional support than on wellbeing (73\%).

\subsection{Data analysis}

Qualitative data was extracted from questionnaires onto a spread sheet and the extraction process independently checked. A framework approach [34] was used to systematically code, classify and organise data into key themes with due attention to rigour. Extracts were read repeatedly by RH and SM to identify recurring concepts and categories which formed the basis of a framework to code and classify data, reflecting the content and issues expressed by respondents. Initial coded categories and themes were then sorted and grouped into higher order themes based on similarity of content. RH and SM worked independently, following which a final consensus was achieved, removing any ambiguity and overlap. FK independently checked the data extraction process for accuracy and reviewed the thematic analysis. As shown in the results below, eight themes were identified; five contributing to both well-being and emotional support (professional expertise and approach, service accessibility, caring attitudes, coping with anxiety and depression, changing healthcare needs); one to well-being alone (medical liaison and referral) and two to emotional support alone (family emotional support; emotional support not needed or provided).

\section{Results}

\subsection{Theme 1: Professional Expertise and Approach}

Many respondents commented positively on the professional qualifications, breadth of specialist knowledge and expertise of the ACHD nursing team, which inspired feelings of confidence, provided reassurance about their medical condition on an individual basis and contributed positively to both well-being and emotional support. 
"All the team are very well qualified and always were happy to help me."

“...(referring to dimensions of care). Physically, psychologically and emotionally they are reliable and informed and highly professional. The service offered is exceptional."

"Having access to expertise and advice enables one to feel more confident and informed."

"They are reassuring and have specialist knowledge of my condition."

Others cited contributions to well-being and emotional support made through creation of positive therapeutic relationships and benefits of individualised approaches to care which made respondents feel they were not just a number on the in-patient list. Professionalism maintained when working pressures on the service were substantive was appreciated by respondents.

"Over the course of my health issue this year, I built up a good relationship with one of the (ACHD) nurses and this was appreciated and helpful as I didn't need to re-explain my issues. I received very professional and individualised care. Being a nurse myself I was impressed as I know how pressurised nurses are." "It really made a big impact on me when (named nurse) came to visit me in the ward. It made me feel like a person, not a number and really showed how professional they are."

\subsection{Theme 2: Service Accessibility}

Many respondents identified the telephone accessibility of the ACHD nursing service as a major positive influence on both mental well-being and emotional support. This accessibility offered patients a chance to discuss any immediate concerns, enhancing reassurance and reducing post-discharge feelings of isolation. For some, the fact that it was possible to speak to a specialist nurse without having to wait to speak to medical staff was perceived as a further benefit.

"I feel it is very reassuring to know that the (ACHD) team are at the end of a 'phone if I have any concerns or questions and are (sic) never made to feel that I am being a bother to them. They are always so helpful and supportive."

"Knowing that I can contact the team has made me feel that I am not alone and help is available."

"Mental well-being is very important. To know that you can call a specialist nursing team without having to speak to doctors is very good."

\subsection{Theme 3: Caring Attitudes}

Respondents cited many caring attitudes and skills which they felt contributed to both well-being and emotional support. Listening skills were particularly valued as was providing advice in a non-judgemental way together with the holistic approach to care of the individual, which made them feel valued.

"They are supportive and any emotional support I need they will listen and not judge me in any way."

"Demonstrate a very caring attitude to help the whole person, medical, physical and emotional."

In addition to listening and helping skills, many cited kindness and positive attitudes such as friendliness as influential on both well-being and emotions.

"If it wasn't for their kindness, positive attitudes and knowledge I wouldn't be here to tell my story."

"A friendly face and a warm smile followed by a listening ear and helpful advice is always given."

For some patients, empathetic approaches, listening skills and practical support through stressful life events such as grieving after parental loss were important.

“... when my father died suddenly, the nurses, especially (named nurse) really did all they could and still do every time I go to hospital, they really listen and really try and help."

\subsection{Theme 4: Coping with Anxiety and Depression}

Many respondents experienced anxiety pre-operatively and found that information provided by specialist nurses allayed concerns, reducing their anxiety. Visits by the specialist nurse to the intensive care unit (ICU) while in the post-operative period were also helpful in reducing anxiety, as was home follow-up contact by email and 
'phone.

"I was very anxious pre-operation (named nurse) reassured me of my concerns during a pre-operation visit to the ward/ICU and also visited me in ICU to again offer support and reassurance."

"(Named nurses) provided first class attention and information and reassurance prior to surgery -at a time when I was very anxious."

"They emailed and called me after my operations-were always there to talk to if I was worried about something."

In the longer term, some respondents experienced depression and appreciated specialist nurse referral to a clinical psychologist. Others found annual monitoring visits particularly reassuring, since they offered the opportunity to resolve any health issues before cardiac complications occurred. Reassurance by nurses was also found to be helpful for problems which were recognised to be beyond the expertise of the General Practitioner (GP), but not requiring the intervention of a hospital consultant.

"A few months ago I was feeling low and depressed. The (ACHD) nurse I spoke to totally understood how I was feeling. She dealt with my feelings and put me in touch with the right person."

"Yearly visit is a reassurance to me, as I know I can sort out any problems before my health gets affected and prevents my heart condition from getting worse."

"Reassurance when needed for smaller issues that are beyond the GP, but not so important for the consultant to spend time on."

Most respondents found provision of information reassuring and helpful, notably in accessing support, although one noted that stressed and depressed patients might not be capable of contacting the service in this way. Usage of language intended by professionals as supportive could be a source of anxiety. Others noted the long-term emotional problems which could persist years after surgery, for which they said counselling help was needed.

"Information in terms of contact numbers for help given. However, I think those people who may be depressed or stressed might not take the initiative to get in touch."

"...when they say my condition is rare/unique, it makes me worried and scared (although the doctors are guilty of this as well)."

"I should be offered counselling as still emotionally going through some stuff regarding heart operation eleven years ago."

\subsection{Theme 5: Changing Healthcare Needs}

For some respondents, the important nursing interventions to foster well-being and emotional support related to adjustment to events across the lifespan. Others identified support provided for changing needs over the perioperative surgery trajectory, or for having cardiac assistive devices implanted, as important.

"My needs have changed since birth. The onset of 'GUCH' made such a difference especially as a teenager and young adult, then mother,"

"They help you right from the start. From pre-admission, on the ward and as an outpatient."

"After having an ICD (implantable cardioverter defibrillator) implanted, the nurses were very supportive and gave me lots of booklets to read as well as outside support groups to contact."

\subsection{Theme 6: Medical Liaison and Referral}

Findings within this theme contributed positively to well-being alone. For many, rapid referral via the ACHD nursing service for a medical opinion if problems arose offered feelings of security when living with a long-term condition. Others described the value of having access to a service which could achieve liaison with medical staff across diverse organisational boundaries without giving rise to concerns that they were causing undue "hassle".

"Security of knowing someone who understands my condition and my medical history is there and can refer me immediately to an (ACHD) doctor if a problem occurs." 
"The (ACHD) service is a link between patients and doctors. Without them it would be very difficult to liaise with other specialist services and departments. We need their support and expertise to enable referrals from other departments/hospitals to reach our cardiologists."

"They have generally been there when needed and are a welcome link within the medical context to the clinicians and treatments. Someone to contact without feeling (you are) hassling others who already have enough to do treating us. They make time to chat about your cat or your flat as well as what a supraventricular ectopic is."

\subsection{Theme 7: Family Emotional Support}

Respondents appreciated not only the emotional support that specialist nurses gave to them, but also to their families. This extended in one case to telephone contact by the specialist nurse during a patient's critical care admission at another hospital, which had been a source of comfort and support to the family.

"An (ACHD) nurse rang our local hospital when I was in Intensive Care and the support this gave my family was tremendous."

"The specialist nurse who supervises my care has been of great emotional support to me and my family."

Others experienced positive family support in the acute perioperative stages of treatment, but also drew attention to the longer term psychosocial, mobility and economic problems which required resolution and continuation of family support.

"As a family we were given some emotional support, before, during and immediately after the op' but not lately, 8 - 10 months on there are still some issues to resolve — confidence, stress, headaches, employment, travel issues...”

\subsection{Theme 8: Emotional Support Not Needed or Provided}

A minority of respondents stated either they did not need emotional support from the specialist nurses, or did not receive it or know about the service. One felt very strongly that emotional support was beyond the nursing remit and another that doctors and nurses did not have insights into the patient experience.

"Though I have not needed emotional support as such, the nurses always have time to discuss any issues and am sure would have time to help emotionally."

"I did not have any support emotionally after my operation. I did not know about (ACHD) nurses and who they were when I was admitted to hospital."

"This is really outside the nurses remit. (Named nurse) does what she can and is very sympathetic and caring but cannot really provide or isn't in a position to provide emotional support. It's an unfair question."

"I don't seek emotional support from doctors and nurses. They have no idea what patients are experiencing."

\section{Discussion}

Findings demonstrated that a specialist ACHD nursing service made an important, positive contribution to patient well-being and emotional support. These findings are in agreement with quantitative findings indicating substantive satisfaction with these elements of care [33]. However, some limitations are acknowledged: survey response bias cannot be excluded, qualitative findings cannot be generalised and the service setting, structure and specialist role function may not be representative of others. Notwithstanding this, insights have been gained regarding the impact of this specialist service, which contribute to the sparse nursing literature and can form a basis for service development and further investigation.

Findings show that specialist nursing knowledge and expertise which enabled the delivery of a service characterised by individualised and holistic care and reassuring therapeutic relationships, gave patients confidence, contributing positively to both well-being and emotional support. Furthermore, caring attributes which encompassed listening in a non-judgemental way, kindness, and warmth were also identified as contributing positively to patient well-being and emotional support. These findings are congruent with others which have identified holistic approaches, emotional support and caring attributes of specialist and advanced practitioners as contributing 
highly to service user satisfaction, but not specifically to well-being [27] [28].

Other contributions to emotional support and well-being were in enabling patients to cope with anxiety and depression, acknowledged to be important in this diagnostic group with a long-term condition [18]-[20]. Specialist nursing interventions reduced anxiety prior to and following surgery by providing information and reassurance, proactively contacting patients following discharge home and referring individuals with more enduring depression to a clinical psychology service. Accessibility of the service via telephone also allayed many patients concerns and provided reassurance. Similar findings have been reported in other studies highlighting the importance of access, referral and provision of timely information by specialist nurses [29] [30], and also relevant to informational continuity of care [30]-[32]. Meeting patients changing needs across the lifespan and during the hospital-discharge trajectory also contributed to emotional support and well-being. It has been recognised that the increasing life expectancy of ACHD patients [1]-[7] will require services to adjust to the needs of older patients, which has implications for the continuing professional development necessary to equip nurses with enhanced specialist skills [13]. More widely, adjusting care to meet changing needs can contribute to flexible and long-term continuity of care [30]-[32] and this may underpin selective effects on well-being and emotional support reported in this paper.

Findings also highlighted the important contribution to patient well-being made by liaison with medical staff, mediated by increased feelings of security. Other studies have noted the importance of specialist nurses acting as an intermediary with medical and other staff [29], thereby contributing to cross boundary continuity of care. Family support was also a powerful sole contributor to emotional support which has been noted in other investigations of cardiac patients, documenting effects of ill health on the whole family and the importance of nursing interventions to support family functioning [35].

\section{Conclusions and Recommendations}

Professional knowledge and expertise, service accessibility, caring attributes and enabling patients to cope with anxiety and depression contributed positively to well-being and emotional support of ACHD patients. Family support was influential on emotional support and medical liaison exerted an impact on well-being by enhancing feelings of security. Meeting changing needs also influenced well-being and emotional support positively. The ACHD nursing service contribution to well-being and emotional support also underpins elements of continuity of care. At a time when specialist roles and services are under review [13], it is recommended that contributions to well-being and emotional support inform future specialist care and service delivery and that periodic re-evaluations including patient's perspectives are conducted after any service changes. Future demand for emotional support services (clinical psychology, counselling) also need consideration in service review. A minority of patients did not receive information about the specialist nursing service or understand its purpose in terms of medical and nursing roles; this should prompt a review of communication approaches.

\section{Acknowledgements}

The authors acknowledge with gratitude the patients who contributed to this study and to London South Bank University for funding a research bursary for Dr. Richard Hatchett.

\section{References}

[1] Marelli, A.J., Mackie, A.S., Ionescu-Ittu, R., Rahme, E. and Pilote, L. (2007) Congenital Heart Disease in the General Population: Changing Prevalence and Age Distribution. Circulation, 115, 163-172.

http://dx.doi.org/10.1161/CIRCULATIONAHA.106.627224

[2] Verhengt, C.L., Uiterwaal, C.S.P.M., Van der Velde, E.T., Pieper, P.G., Van Dijk, A.P.J., Vliegen, H.W., Grobbee, D.E. and Mulder, B.J.M. (2010) Mortality in Adult Congenital Heart Disease. European Heart Journal, 31, 1220-1229. http://dx.doi.org/10.1093/eurheartj/ehq032

[3] Grobbee, D.E., Mulder, B.J., Uiterwaal, C.S.P.M., Meijboom, F.J., Plokker, H.W., Van Der Velde, E.T., Verhengt, C.L., Pieper, P.G. and Sieswerda, G.T. (2010) Emerging Burden of Hospital Admission of Adults with CHD. Heart, 96, 872-878. http://dx.doi.org/10.1136/hrt.2009.185595

[4] Negishi, J., Ohuchi, H., Yasuda, K., Miyazaki, A., Norifumi, N. and Yamada, O. (2015) Unscheduled Hospital Admission in Adults with CHD. Korean Circulatory Journal, 45, 59-66. http://dx.doi.org/10.4070/kcj.2015.45.1.59

[5] British Cardiac Society (2002) Grown Up Congenital Heart (GUCH) Disease: Current Needs and Provision of Services 
for Adolescents and Adults with Congenital Heart Disease in the UK. A Report of the British Cardiac Society Working Party. Heart, 88, 1-14.

[6] Department of Health (2006) Adult Congenital Heart Disease: A Commissioning Guide for Services for Young People and Grown Ups with Congenital Heart Disease (GUCH). HMSO, London.

[7] British Heart Foundation (2010) Transparency in Outcomes: A Framework for the NHS. http://www.bhf.org.uk

[8] Moons, P. (2005) Nurse Specialists in Adult Congenital Heart Disease: The Current Status in Europe. European Journal of Cardiovascular Nursing, 5, 60-67. http://dx.doi.org/10.1016/j.ejcnurse.2005.10.010

[9] Moons, P., Canobbio, M.M. and Harrison, J. (2006) The International Adult CHD Network: Coming Together for the Future. Progress in Cardiovascular Nursing, 21, 94-96. http://dx.doi.org/10.1111/j.0889-7204.2006.04971.x

[10] Vernon, S., Finch, M. and Lyon, J. (2011) Adult Congenital Heart Disease: The Nurse Specialists Role. British Journal of Cardiac Nursing, 6, 88-91. http://dx.doi.org/10.12968/bjca.2011.6.2.88

[11] Ladouceur, M., Iserin, L., Cohen, S., Legendre, A., Boudjemline, Y. and Bonnet, D. (2013) Key Issues of Daily Life in Adults with Congenital Heart Disease. Archives of Cardiovascular Diseases, 106, 404-412. http://dx.doi.org/10.1016/j.acvd.2013.02.004

[12] Leary, A. and Oliver, S. (2010) Clinical Nurse Specialist: Adding Value to Care: An Executive Summary. Royal College of Nursing Publications, London.

[13] Griffiths, L., Habibi, H., Kennedy, F., Lyon, J., Quirk, J. and Vernon, S. (2015) ACHD Nursing: Guidance on Roles, Career Pathways and Competence Development. Royal College of Nursing Publications, London.

[14] The Kings Fund London: Point of Care Programme (2013) http://www.kingsfund.org.uk/projects/point-of-care/about

[15] Kamphuis, M., Verloove-Van Horich, S.P., Ottenkamp, J., Vliegen, H.W. and Vogels, T. (2002) Disease Related Difficulties and Satisfaction with Levels of Knowledge in Adults with Mild or Complex Congenital Heart Disease. Cardiology in the Young, 12, 266-271. http://dx.doi.org/10.1017/S1047951102000574

[16] Moons, P., Van Deyk, K., Marquet, K., De Bleser, L. and Budts, W. (2009) Profile of Adults with Congenital Heart Disease Having a Good, Moderate or Poor Quality of Life: A Cluster Analytic Study. European Journal of Cardiovascular Nursing, 8, 151-157. http://dx.doi.org/10.1016/j.ejcnurse.2008.11.001

[17] Vigl, M., Niggemayer, E., Hager, A., Schwedler, G., Kropf, S. and Bauer, U. (2011) The Importance of Socio-Demographic Factors for Quality of Life of Adults with Congenital Heart Disease. Quality of Life Research, 20, 169-177. http://dx.doi.org/10.1007/s11136-010-9741-2

[18] Van Rijen, E.H.M., Utens, E.M.W.J., Roos-Hesselink, J.W., Meijboom, F.J., Van Domburg, R.T., Roelandt, J.R.T.C., Bogers, A.J.J.C. and Verhulst, F.C. (2004) Medical Predictors for Psychopathology in Adults with Operated Congenital Heart Disease. European Heart Journal, 25, 1605-1613.

[19] Bromberg, J.I., Beasley, P.J., D’Angelo, E.J. and Landzberg, M. (2003) Depression and Anxiety in Adults with Congenital Heart Disease: A Pilot Study. Heart Lung, 32, 105-110. http://dx.doi.org/10.1067/mhl.2003.26

[20] Eslami, B., Sundin, O., Macassa, G., Khankeh, H.R. and Soares, J.J. (2013) Anxiety, Depressive and Somatic Symptoms in Adults with Congenital Heart Disease. Journal of Psychosomatic Research, 74, 49-56. http://dx.doi.org/10.1016/j.jpsychores.2012.10.006

[21] Centres for Disease Control and Prevention (2013) Health Related Quality of Life. Well-Being Concepts. http://www.cdc.gov/hrqol/wellbeing.htm

[22] Dodge, R., Daly, A.P., Huyton, J. and Sanders, L.D. (2013) The Challenge of Defining Well-Being. International Journal of Wellbeing, 2, 222-235. http://dx.doi.org/10.5502/ijw.v2i3.4

[23] Cruickshank, S., Kennedy, C., Lockhart, K., Dosser, I. and Dallas, L. (2008) Specialist Breast Care Nurses for Supportive Care of Women with Breast Cancer. Cochrane Database of Systematic Reviews, No. 1, Article No. CD005634. http://dx.doi.org/10.1002/14651858.cd005634.pub2

[24] Lloyd Jones, M. (2005) Role Development and Effective Practice in Specialist and Advanced Practice Roles in Acute Hospital Settings: Systematic Review and Meta-Synthesis. Journal of Advanced Nursing, 49, 191-209. http://dx.doi.org/10.1111/j.1365-2648.2004.03279.x

[25] Loveman, E., Royle, P. and Waugh, N. (2003) Specialist Nurses in Diabetes Mellitus. Cochrane Database of Systematic Reviews, No. 2, Article No. CD003286. http://dx.doi.org/10.1002/14651858.cd003286

[26] Belling, R., McLaren, S. and Woods, L.P. (2009) Specialist Nursing Interventions for Inflammatory Bowel Disease. Cochrane Database of Systematic Reviews, No. 4, Article No. CD006597. http://dx.doi.org/10.1002/14651858.cd006597.pub2

[27] Begley, C., Elliott, N., Lalor, J., Coyne, I., Higgins, A. and Comiskey, C.M. (2013) Differences between Clinical Specialist and Advanced Practitioner Clinical Practice, Leadership, and Research Roles, Responsibilities, and Perceived 
Outcomes (The SCAPE Study). Journal of Advanced Nursing, 69, 1323-1337. http://dx.doi.org/10.1111/j.1365-2648.2012.06124.x

[28] Begley, C., Murphy, K., Higgins, A., Elliott, N., Lalor, J., Sheerin, F., et al. (2010) Evaluation of Clinical Nurse and Midwife Specialist and Advanced Nurse and Midwife Practitioner Roles in Ireland (SCAPE) Final Report. National Council for the Professional Development of Nursing and Midwifery in Ireland, Dublin.

[29] Corner, J., Halliday, D., Haviland, J., Douglas, H.R., Bath, P., Clark, D., et al. (2003) Exploring Nursing Outcomes for Patients with Advanced Cancer Following Intervention by Macmillan Specialist Palliative Care Nurses. Journal of Advanced Nursing, 41, 561-574. http://dx.doi.org/10.1046/j.1365-2648.2003.02568.x

[30] Aspinal, F., Gridley, K., Bernard, S. and Parker, G. (2012) Promoting Continuity of Care for People with Long-Term Neurological Conditions: The Role of the Neurology Nurse Specialist. Journal of Advanced Nursing, 68, 2309-2319. http://dx.doi.org/10.1111/j.1365-2648.2011.05928.x

[31] Waibel, S., Henao, D., Aller, M.B., Vargas, I. and Vázquez, M.L. (2012) What Do We Know about Patients’ Perceptions of Continuity of Care? A Meta-Synthesis of Qualitative Studies. International Journal for Quality in Health Care, 24, 39-48. http://dx.doi.org/10.1093/intqhc/mzr068

[32] Freeman, G., Shepperd, S., Robinson, I., Ehrich, K. and Richards, S. (2002) Continuity of Care: Report of a Scoping Exercise for the NCCSDO. NCCSDO, London.

[33] Hatchett, R., McLaren, S., Corrigan, P. and Filer, L. (2015) Evaluation of a Specialist Nursing Service for Adult Patients with Congenital Heart Disease. International Journal of Nursing Practice, 21, 556-565.

[34] Ritchie, J. and Spencer, L. (1994) Qualitative Data Analysis for Applied Policy Research. In: Bryman, A., Burgess, R.G., Eds., Analyzing Qualitative Data, Routledge, New York, 173-194. http://dx.doi.org/10.4324/9780203413081_chapter_9

[35] Astedt-Kurki, P., Lehti, K., Tarkka, M.T. and Paavilainen, E. (2004) Determinants of Perceived Health in Families of Patients with Heart Disease. Journal of Advanced Nursing, 48, 115-123. http://dx.doi.org/10.1111/j.1365-2648.2004.03178.x 